\title{
LA CULTURA Y EL ARTE ITALIANOS EN LA OBRA DE HENRY JAMES
}

$M^{*}$ Antonia Alvarez Calleja

UNED

En estos últimos años se han prodigado tanto -en cantidad y en calidad- los estudios sobre la obra de Henry James, que puede resultar arriesgado cualquier nuevo intento. No obstante, hay aún ciertos temas que quizá no hayan sido investigados suficientemente, como puede ser la aparición de continuas referencias a la cultura y al arte italianos a lo largo de toda su obra, y la clara influencia que Italia ejerció sobre él. Ya en su ensayo "Gabriele D'Annunzio" (1904) confesaba:

"I firmly believe that the greatest sum of future dominion will precisely be that which shall have its apex in Rome", such being the confidence of the "Latin" spirit. And when the Latin spirit, after many disadventures, clears itself we shall see how all the treasure has been in its keeping.

A pesar de que incluso en las historias americanas sea continua esta presencia de la cultura y el arte italianos en la obra de James, su visión de Italia fue cambiando progresivamente para hacerse cada día más profunda: en los primeros apuntes de viajes se interesa principalmente por la pintura, la arquitectura o el paisaje; en su fase intermedia se concentra más en las costumbres, en la forma de ser y comienza a analizar el conflicto que se produce entre esas dos formas diferentes de civilización -la del Viejo y la del Nuevo Mundo- que va a hacer sucumbir a la mayoría de sus personajes americanos, y en sus últimas 
obras logra llegar a un "consenso social" entre las mentalidades opuestas de los dos continentes, debido a sus relaciones cada día más intimas, pero también deja vislumbrar lo que sus libros de notas y cartas ya venian apuntando, la desaparición de todo lo que había tenido significado para su concepto de civilización occidental.

El tratamiento que concede James al tema América versus Europa descubre las dos facetas de su propia relación personal con ambos continentes: por un lado, el compromiso del expatriado con las formas de pensar y sentir americanas y, por otro, la atracción tan fuerte que Europa ejercía sobre él, hasta el punto de hacerle fijar definitivamente su residencia en este lado del Atlántico.

Henry James puede considerarse posiblemente el primer escritor cosmopolita y esto resulta esencial para la riqueza de su narrativa. La visión que la anterior generación de escritores -Washington Irving, Longfellow, Cooper o Hawthorne- tenía de Europa estaba llena de los prejuicios típicamente americanos, y James se distingue de sus predecesores en que comienza en seguida a analizar sus propias experiencias y a sacar sus propias conclusiones, estableciendo la oposición inocencia-experiencia, conciencia-tradición, moral-costumbres y AméricaEuropa. Estaba convencido de que el Nuevo Mundo era moralmente más sano que el Viejo y que esta diferencia de valores morales venía dada por el complejo desarrollo histórico de las dos civilizaciones.

Por otro lado, la civilización italiana ofrecía dos posibilidades diferentes a los personajes americanos de James: en el mejor de los casos, cultura y libertad -como Mary Garland o Maggie Verver- y, en el peor, fracaso y reclusión ${ }^{1}$, llegando a sucumbir muchos de ellos tanto a causa de su propia incapacidad para resistirlos -como Roderick Hudson- o por culpa de los otros -como Daisy Miller, Milly Theale o Isabel Archer.

La dicotomía América-hebraísmo y Europa-helenismo constituía su ideal de civilización ${ }^{2}$ : la integridad moral de Nueva Inglaterra unida a la condición estética que ofrecía Italia eran las dos cualidades que había de poseer ese ser civilizado por excelencia. Por eso, le escribía a su hermano William en 1870.

If I keep along in Italy patiently I rather think I shall become a great man.

Michael Swam, "Henry James", en el Suplemento a British Book News, London, 1950, pp. 13-14

2 Alwyn Berand, Culture and Conduct in the Novels of Henry James, Cambridge, Press Syndicate of the University, 1981, p. 83. 
Esto explica lo que Italia significaba para él y por qué necesitaba la atmósfera italiana como inspiración para sus escritos. Es cierto que James había empezado sus contactos con Italia desde niño, debido a las ideas de su padre de que sus hijos recibieran una educación europea, pero lo que verdaderamente le descubrió las posibilidades de Italia fue su primer viaje de "iniciación" ya adulto. Cuando llego a Roma en 1869 , mientras paseaba por sus plazas, sus ruinas y sus monumentos, supo lo que el arte y la cultura italianos significarían para él en el futuro:

...here I am then in the Eternal City. At last -for the first time- I live! It beats everything: it leaves the Rome of your fancy -your educationnowhere... I shall go to bed a wiser man (Letters, 25).

Durante muchos años el hechizo de Italia permaneció tan fuerte dentro de él que la mayoría de las historias que nacen de sus primeros viajes a Europa las sitúa en Italia; son una especie de relato dramatizado de su viaje a Italia en $1873^{3}$, cuando tuvo ocasión de reunirse con los artistas americanos instalados en Roma en suntuosos palacios y estudios pintorescos. El pequeño y compacto grupo de artistas y aficionados de la época de Hawthorne había aumentado, pues los artífices americanos continuaban acudiendo a Roma por una buena razón: en América no habia escuelas de arte ni grandes maestros con quienes estudiar, ni tampoco un clima adecuado para el creador. Sin embargo, en Italia los jóvenes escultores americanos encontraban el mejor mármol para cincelar sus obras y, al igual que los pintores, podían admirar maravillosos ejemplos y precedentes; para el novelista había temas, mientras que América no ofrecía en su rápida expansión ni misterio ni ruinas del pasado, sino sólo esa prosperidad prosaica producida por la nueva revolución industrial, lo que era a todas luces insuficiente para un novelista de imaginación. Con los artistas llegaban los dilettantes, que proporcionaban una inmensa variedad de tipos, y el mayor número lo formaban los americanos que disfrutaban del clima amable de Roma.

En carta a su madre del otoño de 1869 , James le decía que la colonia americana de Roma era "a very poor affair" y que la única palabra que podía definirles era "vulgar", por su total ignorancia y su

${ }^{3}$ Christof Wegelin, The Image of Europe in Henry James, Dallas, Southern Methodist University Press, 1958, p. 27. 
"stingy, defiant, grudging" actitud hacía cualquier cosa que fuera europea (Letters, 22).

James conocía a muchos artistas americanos en Roma y menciona a algunos de ellos en sus cartas. Estuvo estudiando de cerca una serie de "casos" que incorporó a sus novelas, incluso él mismo era uno de esos artistas, a pesar de que su medio no fuera el mármol o la pintura. Parece haberse planteado la pregunta de qué clase de artista podía ser un americano en un entorno extranjero: perezoso o perseverante, pretencioso o mediocre, imitador o inspirado pero sin profesionalidad. Llegaría el tiempo en que encontraria la respuesta a esas preguntas y lo iría exponiendo en su obra, hasta llegar a constituir uno de sus temas más importantes:

Italia Y El ARTista Americano

Como representantes más destacadas de este tema hay tres obras fundamentales: The Madonna of the Future, la parábola del pintor americano en Florencia; Roderick Hudson, la historia del escultor genial que sucumbe en Roma, y "The Aspern Papers", la evocación del poeta venerado por su amante veneciana.

En The Madonna of the Future, nos presenta al artista impotente, que pasa su vida en una charla incesante describiendo la Madonna que va a pintar, pero cuando muere no deja más que un lienzo en blanco, ya agrietado y descolorido por el tiempo. Otros dos artistas de la colonia americana son el pintor que únicamente posee habilidad manual, y la coleccionista de arte que carece del menor conocimiento artístico.

El tema se refiere a que Italia puede proporcionar un entorno positivo al artista, pero no los dones innatos que debe poseer para ser un gran creador".

En Roderick Hudson, presenta al artista genial, que triunfa nada más llegar a Roma, en contraste con el resto de los artistas de la colonia americana: el profesional oportunista, el de aspiraciones modestas, la pintora amateur son hábiles en su profesión, pero les falta inspiración, son la medianía.

Esta es su primera novela importante, precisamente porque en ella se anticipa todo el mundo literario de James: la relación de Roderick con Mallet es similar a la de Chad con Strether en The Ambassa-

4 Leon Edel, Henry James: The Conquest of London, 1870-1881, New York, J.B. Lippincott Company, 1962, p. 37. 
dors, Christine Light es antecedente de Madame Merle en The Portrait of a Lady, Kate Croy en The Wings of the Dove, y Charlotte en The Golden Bowl' .

La descripción que nos ofrece de Roma es una de las partes más brillantes: la antigua ciudad imperial y papal proporciona a Hudson el entorno que necesita -obras de arte de grandes maestros como Miguel Angel, un estudio en un barrio pintoresco, mármol de Carrara- en contraste con las limitaciones de su pequeña ciudad de Massachusett.

El mayor contraste se produce en la pareja central: Roderick no sabe vencer los peligros que encuentra en una sociedad con experiencia de siglos y sucumbe. En cambio su prometida Mary Garland, la joven americana, sabe aunar el sentido estético que la brinda Italia con su integridad moral, lo que la eleva al ideal de James: el espíritu culto y civilizado a que aspiran todos sus personajes.

"The Aspern Papers" son las cartas amorosas que un famoso poeta americano escribió en Venecia a su musa, y en este poeta James se evoca a sí mismo como era, o mejor, como le hubiera gustado ser.

Este es uno de los mejores ejemplos de novela corta de toda la literatura, ya que la escribió en la cúspide de su vida artística, 1888. Lo que se denomina su "época mayor" es más elaborada o preciosista, y ésta es principalmente concisa y sutil.

La oposición "presente", "pasado" domina toda la obra: la amante anciana rememora su antigua pasión a través de las cartas que conserva como su mayor tesoro; el diletante americano quiere rastrear las huellas del pasado en la vida del poeta que tanto admira y llega a extremos insospechados por conseguir esas cartas pero no es capaz de dar el paso final; ante la idea de tener que aceptar al mismo tiempo a la vulgar poseedora, retrocede a su mundo moral, lo que es muy característico en los héroes de James, entroncado con el famoso sentido Puritano del deber.

Lo más delicado de esta obra maestra es el monólogo interior del narrador-protagonista, después de huir derrotado por no haber podido conseguir su propósito. Según le va llevando el gondolero a través de los canales venecianos, la visión de la ciudad decadente se funde con la profundidad de sus sentimientos ante su desastre personal, en la más brillante demostración de su técnica conocida por "stream-of-consciousness", desarrollada posteriormente por la siguiente generación de escritores como Virginia Woolf, Proust, Joyce y Faulkner.

Otro tema igualmente importante de la obra de James es el que protagoniza la joven americana independiente y segura de sí misma, que se convierte en el símbolo de las cualidades positivas de América:

5 Edward Wagenknecht, The Novels of Henry James, New York. Frederick Ungar Publishing Co., 1982, p. 36. 
moral, espontaneidad y delicadeza. El antecedente es Minny Temple, su prima y quizá único y verdadero amor de James, que murió a los veinticinco años, y a la que dedicó en sus cartas adjetivos como "espíritu divino e inquieto", "una experiencia de la naturaleza", "heredera de todos los tiempos" o "la heroína de la escena":

\section{ItAlia Y La JOVEn AMERICANA}

Entre tantas otras, tres obras inolvidables de James analizan el conflicto que se produce al no querer renunciar la joven a su herencia americana, como han hecho los expatriados que encuentra en Italia. Henry James es el descubridor de la muchacha americana como tipo y fenómeno social, muy superior al que presentaron sus antecesores, principalmente Hawthorne.

1) Isabel Archer, en The Portrait of a Lady, es su más brillante representación de la "American girl". Está deseosa de saber, de vivir, de correr tras los más altos ideales, pero sucumbe ante lo vulgar y corrupto de la sociedad europea. Al final, después del sufrimiento, se transforma en la "American lady" que afronta su destino, llegando a realizarse totalmente.

Isabel contrasta con el otro personaje central, el cínico y presuntuoso Gilbert Osmond, un americano expatriado en Italia, y pocos villanos psicológicos han sido nunca mejor dibujados ${ }^{6}$. A su vez Osmond contrasta con el vigoroso hombre de negocios americano, espontáneo, natural, sencillo, insensible al arte, pretendiente de Isabel desde el principio de la obra, y a quien ella rechaza una y otra vez, rechazando en su persona a América, por creer que la van a privar de su libertad.

Florencia y Roma están retratadas con toda minuciosidad: sus monumentos, sus museos, la maravillosa "campagna" italiana.

En 1877 James había estado en Florencia, desde donde escribió un artículo para el Atlantic'. Se había reunido con Frank Boott y su hija Lizzie, una pareja simpática, sencilla, incluso conmovedora, que vivian en una colina de Florencia: el padre anciano con su hija, la villa florentina y la campiña italiana, todo esto atrajo la imaginación de James y posteriormente se convirtieron en Gilbert Osmond y su hija

6 Glenda Leeming, Who's who in Henry James, London, Elm Tree Books, Hamish Hamilton, 1976, p. 120.

7 Leon Edel, Henry James: The Conquest of London, 1870-1881, New York, J.B. Lippincott Company, 1962, p. 296. 
Pansy. No se parecían en absoluto a los modelos, pero la imagen de la villa y de la pareja que vivía en ella iba a satisfacer las necesidades de la novela que estaba formándose en su mente y que comenzaría a escribir cerca de ese mismo lugar.

Las diversas mansiones en que se desarrolla la acción tienen un carácter simbólico y contrastan entre sí: la villa florentina de Osmond tiene la fachada como una máscara que reflejara la personalidad del dueño:

...antique, solid, weather-worn, yet imposing front had a somewhat incomunicative character. It had heavy lids, but no eyes (p. 244).

Y el palacio romano, cerrado y oscuro donde vive el matrimonio, representa una auténtica cárcel para Isabel. Ambas contrastan con Gardencourt, en la que James hace un emocionado tributo a la "English country house", por su enorme significado cultural y de refinamiento ${ }^{8}$. Es en ella donde Isabel acarició sus más bellos sueños de libertad.

En esta novela James considera Italia como un gran almacén ${ }^{9}$ lleno de antiguos objetos de arte y Osmond hace de su vida un arte, intentando incluso moldear a su hija Pansy, dentro de su visión artística que coincide con un deseo de dominio.

Para James, los lugares artísticos emanan un influjo como de encantamiento sobre el visitante: es muy significativo que la propuesta de matrimonio de Osmond a Isabel tenga lugar en la basílica de San Pedro. La atmósfera encantada del principal templo de la cristiandad, lleno de objetos de arte e historia, ciega los ojos de Isabel de tal manera que no se da cuenta de que está perdiendo lo que con tanto anhelo deseaba conservar: su libertad.

El final de la novela mantiene un cuidado equilibrio entre forma y sustancia. El boceto trágico se completa al conformarse Isabel con su destino, lo que supone su completa realización. Y en esto hay algo de la idea tradicional del triunfo espiritual del alma por medio de la muerte; una especie de muerte de la carne y de triunfo espiritual ${ }^{10}$, puesto

8 S. Gorley Putt. The Fiction of Henry James, A Reader's Guide to Henry James, Ithaca, New York, Cornell University Press, 1966, p. 130.

9 Laurence B. Holland, The Expense of Vision, Baltimore and London, The Johns Hopkins University Press, 1964 (1982), p. 39.

10 J. A. Ward, "Social Disintegration in The Wings of the Dove", Norton Critical Edition, Ibid., p. 529. 
que James trataba de encarnar en Isabel todas las virtudes de Nueva Inglaterra, contrastadas con el paisaje italiano de Florencia y Roma, cuyas ciudades -tanto su arte como su cultura- James conocía tan bien.

2) En The Wings of the Dove James presenta la más espiritual de sus heroínas, Milly Theale, y la obra está totalmente enmarcada por Venecia. Como ciudad en la que se mezclan las culturas oriental y occidental, Venecia es el escenario perfecto para la encarnación de Milly, la "heredera de todos los tiempos", y como ciudad decadente y muerta, es el marco adecuado para su desaparición. La peculiar geografía cerrada de Venecia la convierte en la mejor imagen del estado mental de claustrofobia del drama, con Milly encerrada y silenciosa en el palacio Lepporelli hasta su muerte.

Este palacio es en realidad el Palazzo Barbaro", construido en el siglo XV, donde James estuvo en 1887 con sus amigos los Curtis. Fue a pasar diez días, pero permaneció durante cinco semanas, la visita privada más larga que haría jamás.

James habia estado en palacios suntuosos, pero nunca había vivido en uno tan principesco. Le encantaba ese palacio tranquilo, con sus mármoles y frescos, y sus retratos de los dogos. Le gustaban principalmente las habitaciones silenciosas y frescas de la parte posterior, que miraban hacia la penumbra de un patio, a través de las ventanas góticas colocadas a niveles totalmente arbitrarios. A él le designaron una habitación para trabajar con un "pomposo" Tiépolo como techo, y con las paredes enteladas de "ancient pale-green damask".

En uno de los pasajes de la novela James describe la plaza de San Marcos: tres lados de la plaza cubiertos de tiendas y el cuarto dominado por la mole de la catedral, llena de "Renaissance paintings and Byzantine mosaics, and with the bread and wine sacred to the claims of Christian communions". Para James la plaza de San Marcos era como "a greal social saloom, a smooth-floored, blue-roofed chamber of amenity" (p. 288).

Su concepto de Venecia cambió desde que en 1894 su gran amiga Fenimore Woolson se suicidó aquí, considerándola a partir de entonces "obscured" y "saddened" por ese motivo'

En The Wings of the Dove Venecia se adapta tanto a la accion, que incluso cambia cuando aparece la tragedia: de verano luminoso pasa a tormenta, lluvia, oscuridad, cuando Lord Mark -que irónicamente lleva el nombre del Santo Patrono de la ciudad-, le confiesa la sucia intriga tramada contra ella.

\footnotetext{
"Leon Edel, Henry James: The Middle Years, 1882-1895, New York: J.B. Lippincott Company, 1962, p. 227.

12 Ibid, p. 386.
} 
En esta novela James dramatiza la desintegración de la civilización occidental, y lo hace a través de la idea de Milly, que considera que el arte del pasado es irrelevante para la vida moderna ${ }^{12}$.

The Wings of the Dove ha traspasado los límites del realismo y ha llegado a la frontera de lo mítico, y lo mismo ocurre con la ciudad de Venecia, que evoca las asociaciones más familiares, dependiendo el mismo estilo y el tono de la ciudad en su decadencia: la melancólica resonancia de su pérdida se convierte en la verdadera nota de su distinción.

3) En The Golsen Bowl, la "American girl" sufre una transformación: en vez de sucumbir como sus antecesoras, sin abandonar su moral, aunque sí su inocencia, se convierte en la mujer inteligente y apasionada's.

Al contrario de las demás obras, ésta no se desarrolla en Italia. Lo que le interesa aquí a James son las costumbres tradicionales de la aristocracia romana, para contrastarlas con las de América ${ }^{14}$. La figura del Príncipe Amerigo es la expresión más clara de la simpatía que durante toda su vida sintió James por la civilización latina, y supone uno de sus grandes logros: es sensual, joven, viril, incapaz de hipocresía, católico de hecho aunque de sensibilidad pagana; su esteticismo es una forma de su materialismo. Se transforma de modo tan vigoroso su carácter a través de la obra, que aquí el contraste se produce entre el primero y el segundo Amerigo.

James nos hace ver al Príncipe y la civilización que le ha producido en términos estructurales o arquitectónicos, comparándolo con la amplitud de los viejos palacios y los majestuosos jardines. Miles de años y el esfuerzo humano le han moldeado hasta lograr una especie de "heir of the ages":

a beautiful persobal presence, that of a prince in very truth, a ruler, a warrior, patron, lighting up brave architecture... the ghost of some proudest ancestor (p.42).

13 Christof Wegelin. The Image of Europe in Henry James, Dallas, Southern Methodist University Press, 1958, p. 122.

14 Sallie Sears, The Negative Imagination: Form and Perspective in the Novels of Henry James, Ithaca, New York, 1968, p. 198. 
Como Maggie le había definido, Amerigo se asemeja a uno de esos objetos valiosos que su padre ha ido coleccionando en Italia:

"Yo are at any rate a part of his collection, one of the things that can only be got over here. You're a rarity, an object of beauty, an object of price... you belong to a class about which averything is known (p.23).

Las dos figuras femeninas son opuestas: Maggie, la joven americana, encarna todos los valores positivos, y Charlotte, la expatriada, los negativos, aunque James la trate mejor que a otros de sus personajes malvados. Pero la oposición básica de la novela es la lucha entre dos orientaciones de vida, dos actitudes vitales que controlan las respuestas y el comportamiento de los individuos; el contraste está entre el viejo y el nuevo mundo, la Italia clásica y la América moderna. Tanto Maggie como Amerigo están vistos históricamente, son la representación más acabada de sus respectivas civilizaciones.

El tema de The Golden Bowl se sitúa por encima del "internacional" de tantas obras de James. La escena final representa algo más que una "fusión social", es en realidad la unión de dos virtudes: la energía espiritual de Maggie y la "forma" de Amerigo, lo que representa un "sublime consenso" entre el Príncipe italiano Amerigo y la chica americana, ahora transformada en una auténtica mujer ${ }^{15}$. Como explica James en el Prefacio de la New York Edition, la última novela del tríptico que forma su "major phase" es "a moral fable emblematic of the achievement of the international social fusion", que James veía como el resultado final de las relaciones sociales cada día más íntimas entre Europa y América.

James estuvo unos años algo olvidado de la crítica, pero el llamado "resurgimiento de Henry James" ha sido en realidad su descubrimiento como figura literaria mundial, puente entre el movimiento romántico y todo lo que es moderno en el arte literario del siglo $\mathrm{xx}$. Y el secreto de que perdure su fama es muy simple: trató exclusivamente de las tensiones que produce la civilización, escribió del hombre y de la mujer en su lucha por controlar emociones y pasiones dentro de la moral y las costumbres de la sociedad, comprendió el motivo y el comportamiento del ser humano, buscó la belleza en vez de la fealdad, la amabilidad en vez de la crueldad y la paz en vez de la violencia.

15 Leon Edel, Henry James: The Master, 1901-1916, New York, J.B. Lippincott Co., 1972, p. 216. 